\title{
Simulation of Scalar Field Theories with Complex Actions
}

\section{Michael C. Ogilvie*}

Department of Physics, Washington University, St. Louis MO USA

E-mail: mco@wustl.edu

\section{Leandro Medina}

Department of Physics, Washington University, St. Louis MO USA

E-mail: leandro.medina.lvegmail.com

\begin{abstract}
Many scalar field theory models with complex actions are invariant under the antilinear (PT) symmetry operation $L^{*}(-\chi)=L(\chi)$. Models in this class include the $i \phi^{3}$ model, the Bose gas at finite density and Polyakov loop spin models at finite density. This symmetry may be used to obtain a dual representation where weights in the functional integral are real but not necessarily positive. For a subclass of models satisfying a dual positive weight condition, the partition function is manifestly positive. The sign problem is eliminated; such models are easily simulated by a simple local algorithm in any number of dimensions. Simulations of models in this subclass show a rich set of behaviors. Propagators may exhibit damped oscillations, indicating a clear violation of spectral positivity. Pattern formation may also occur, with both stripe and bubble morphologies possible. The existence of a positive representation is constrained by Lee-Yang zeros: a positive representation cannot exist everywhere in the neighborhood of such a zero. Simulation results raise the possibility that pattern-forming behavior may occur in finite density QCD in the vicinity of the critical line.
\end{abstract}

The 36th Annual International Symposium on Lattice Field Theory - LATTICE2018 22-28 July, 2018

Michigan State University, East Lansing, Michigan, USA.

\footnotetext{
* Speaker.
} 


\section{PT Symmetry and a New Algorithm for the Sign Problem}

The sign problem is known to be extremely difficult in the general case. Within the general class of problems with complex weights, there is a distinguished class of $P T$ symmetric models, which includes many well-known models with sign problems, such as the $i \phi^{3}$ field theory and QCD at finite density. $P T$ symmetry refers to any symmetry under the action of a discreet symmetry like parity combined with complex conjugation. For a single scalar field $\chi$, the action is invariant under

$$
\mathscr{L}(\chi)=\mathscr{L}(-\chi)^{*}
$$

This is referred to as a $P T$ symmetry because in the quantum-mechanical systems in which the symmetry was initially studied, the field $\chi$ is naturally interpreted as a spatial coordinate $x$. In the case of QCD or similar models at finite density, the symmetry is $C K$ where $C$ is charge conjugation and $K$ is complex conjugation. It can be proven that such systems always have real representations [1], and sometimes there are positive representations where all path integral weights are real. In the latter case, the sign problem is completely removed.

The $P T$ symmetry condition can be used to find a natural class of $P T$-symmetric scalar field models for which there exists a representation where all weights are demonstrably positive. The symmetry of $\mathscr{L}$ tells us that its Fourier tranforms with respect to $\chi$ is a real function $\tilde{\mathscr{L}}(\tilde{\chi})$; this property extends to the action and to path integral weights. Using Fourier transforms in the scalar field variables can be understood as a kind of duality transform appropriate for real-valued fields. We take the action to have the form

$$
S(\chi)=\sum_{x}\left[\frac{1}{2}\left(\partial_{\mu} \chi(x)\right)^{2}+V(\chi(x))-i h(x) \chi(x)\right]
$$

where $V(\chi)=V(-\chi)^{*}$. The external field $h(x)$ is site-dependent, and used to deduce correlators for $\chi$ in the new representation. We now rewrite the kinetic and potential terms as Fourier transforms. The single-site kinetic term is easily written as a Fourier transform:

$$
\exp \left[\frac{1}{2}\left(\partial \chi_{x}\right)^{2}\right]=\int d \pi_{\mu}(x) \exp \left[\frac{1}{2} \pi_{\mu}(x)^{2}+i \pi_{\mu}(x) \partial_{\mu} \chi_{x}\right]
$$

The weight term $w(\chi(x)) \equiv \exp [-V(\chi(x))]$ associated with the potential term $V$ can be written as the transform of a real dual weight $\tilde{w}(\tilde{\chi}(x))$. If $\tilde{w}>0$, then we can define the real dual potential $\tilde{V}(\tilde{\chi}) \equiv-\log [\tilde{w}(\tilde{\chi})]$. After a lattice integration by parts, it is straighforward to integrate out $\chi$ to obtain the action

$$
Z=\int \prod_{x} d \pi_{\mu}(x) \exp \left\{-\sum_{x}\left[\frac{1}{2} \pi_{\mu}^{2}(x)+\tilde{V}(\partial \cdot \pi(x)-h(x))\right]\right\} .
$$

which is postive everywhere. In such a representation, standard lattice simulation methods may be used. This representation is local and may be used in any dimension; algorithmic implementation is easy. The extension to multiple scalar fields is trivial. 


\section{Models: positivity violation, pattern formation and the Bose gas}

We have found a rich set of models with two real scalar fields, $\chi$ and $\phi$; the additional field $\phi$ transforms trivially under $P$. We consider three different models. Our first two models have an imaginary term $-i g \phi \chi$ as part of the potential, while the third, the Bose gas at finite density, has an imaginary term in the kinetic part of the action.

Our first model is exactly solvable in any dimension but displays interesting behavior as $g$ is varied $[2,3]$. This imaginary-coupled quadratic (ICQ) model has a potential $V$ of the form $V(\phi, \chi)=m_{\phi}^{2} \phi^{2} / 2+m_{\chi}^{2} \chi^{2} / 2-i g \phi \chi$. The eigenvalues of the mass matrix are given by either two real masses or a complex conjugate pair, as required by the $P T$ symmetry of the model. The dual potential takes the form $\tilde{V}(\phi, \partial \cdot \pi)=m_{\phi}^{2} \phi^{2} / 2+(\partial \cdot \pi-g \phi)^{2} / 2 m_{\chi}^{2}$. In figure 1 , we show results for one-dimensional simulations of the ICQ model in the two different regions. The difference in behavior is striking between the upper curve where there are two real masses, and the lower curve where there is a complex conjugate mass pair. The lines represent the analytical form of the continuum result for the propagators, and the points are from simulations; the error bars on the points are smaller than the points themselves.

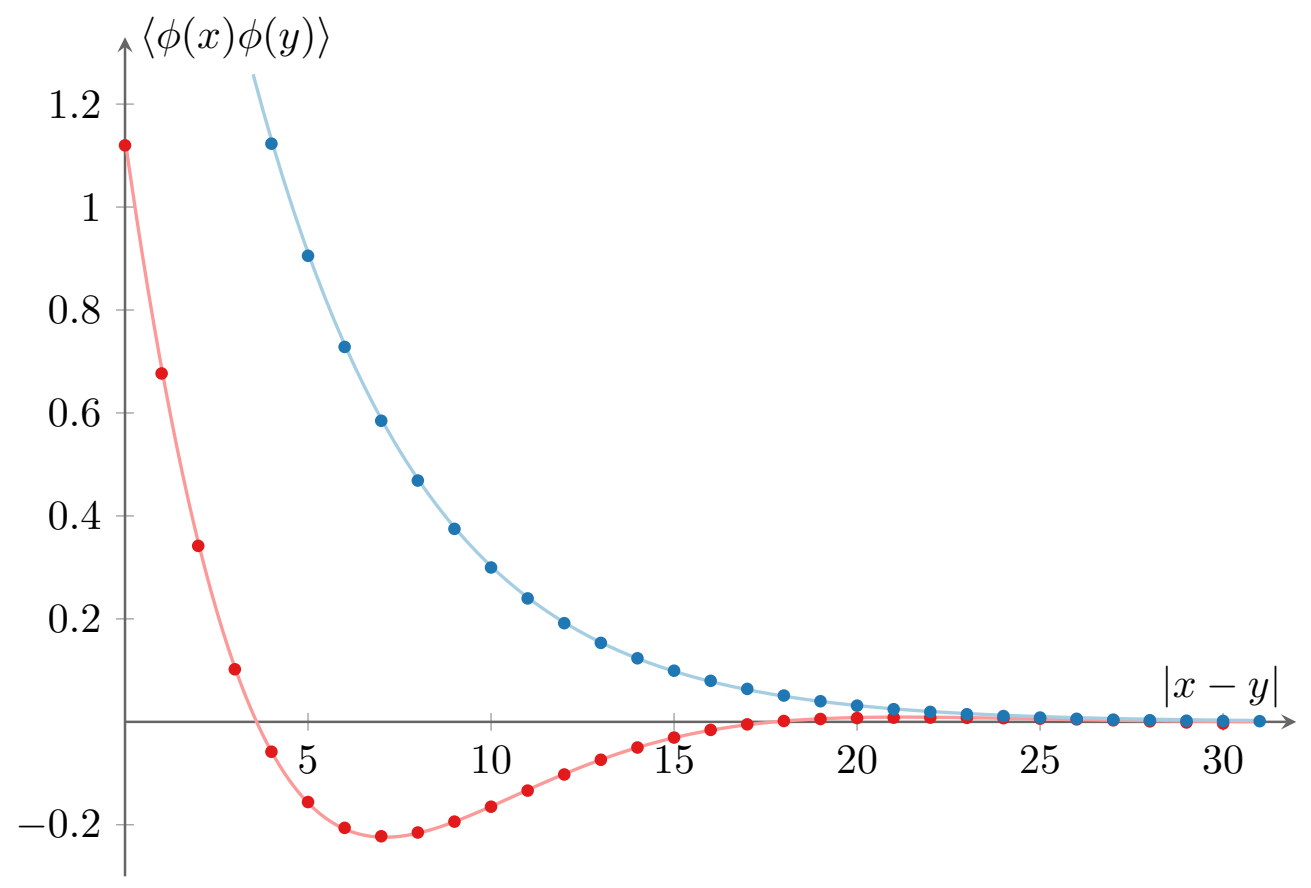

Figure 1: Propagator $\langle\phi(x) \phi(y)\rangle$ as a function of $|x-y|$ for the $d=1$ ICQ model on a lattice with 256 sites. In both curves, $m_{\phi}^{2}=0.001$ and $g=0.1$. The upper (blue) curve corresponds to $m_{\chi}^{2}=0.250$, while the lower (red) curve has $m_{\chi}^{2}=0.002$. The lines represent the analytical form of the continuum result; the errors bars on all points are smaller than the points themselves.

Our second example generalizes the first, with a potential $V(\phi, \chi)=U(\phi)+m_{\chi}^{2} \chi^{2} / 2-i g \chi \phi$ leading to $\tilde{V}(\phi, \partial \cdot \pi)=U(\phi)+(\partial \cdot \pi-g \phi)^{2} / 2 m_{\chi}^{2}$. We consider here the case of the imaginarycoupled double well (ICDW) model, where the potential has the form $U(\phi)=\lambda\left(\phi^{2}-v^{2}\right)^{2}$. Because 


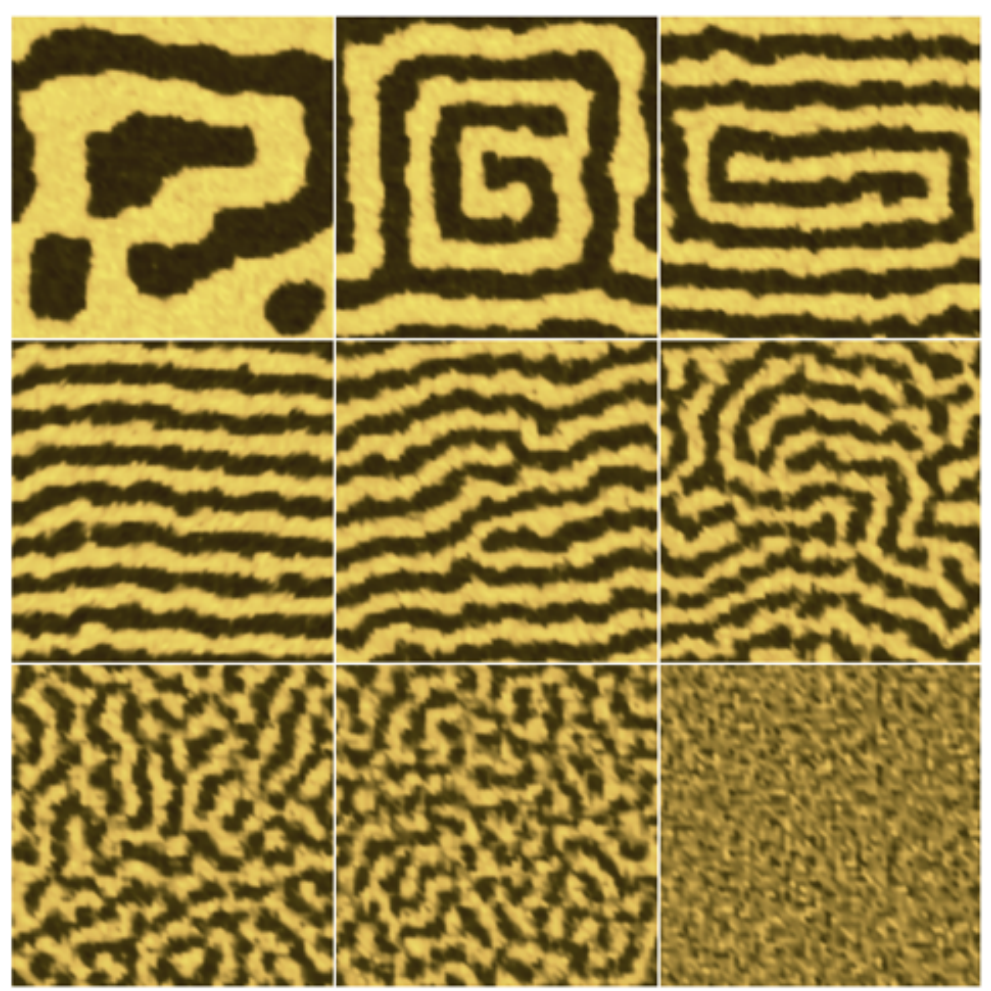

Figure 2: Configuration snapshots of $\phi$ in the $d=2$ ICDW model on a $64^{2}$ lattice for several values of $g$. From left to right, top to bottom: $g=0.8,0.9,1.0,1.1,1.2,1.3,1.4,1.5$ and 2.5. The other parameters are $m_{\chi}^{2}=0.5, \lambda=0.1$ and $v=3$.

the field $\chi$ enters quadratically, it may be integrated out, yielding an effective action of the form

$$
S=\sum_{x}\left[\frac{1}{2}\left(\partial_{\mu} \phi(x)\right)^{2}+U(\phi)\right]+\frac{g^{2}}{2} \sum_{x, y} \phi(x) \Delta(x-y) \phi(x)
$$

where $\Delta(x)$ is a free Euclidean propagator with mass $m_{\chi}$, i.e., a Yukawa potential. This additional term in the action acts to suppress spontaneous symmetry breaking. Models of this type have been used to model a wide variety of physical systems and are known to produce complicated pattern-forming phases $[4,5,6]$. Constant solutions which extremize $S$ can be found by minimizing $U(\phi)+g^{2} \phi^{2} / 2 m_{\chi}^{2}$. Linearizing the equation of motion around such a solution $\phi_{0}$, the inverse propagator is found to be

$$
p^{2}+U^{\prime \prime}\left(\phi_{0}\right)+\frac{g^{2}}{p^{2}+m_{\chi}^{2}}
$$

which is positive near $p^{2}=0$. However, if $U^{\prime \prime}\left(\phi_{0}\right)<0$, the propagator can go negative at larger positive values of $p^{2}$, indicating a tachyonic instability of the homogeneous state. In the region of parameter space where this instability occurs, the equilibrium phase is one where patterns form. This behavior is similar to that found in related condensed matter physics models $[4,5,6]$, as well as in nuclear pasta models $[7,8,9]$. Figure 2 shows configuration snapshots on a $64^{2}$ lattice as $g$ is varied within the tachyonic region. In figure 3 , we show the absolute value of the Fourier transform 
$\tilde{\phi}(k)$ for the same parameters. The ring of enhanced modes, signaled by the appearance of tachyonic modes in the homogenous solution propagator, is striking. This pattern-forming behavior is independent of dimensionality; we have also observed it directly in three-dimensional simulations.

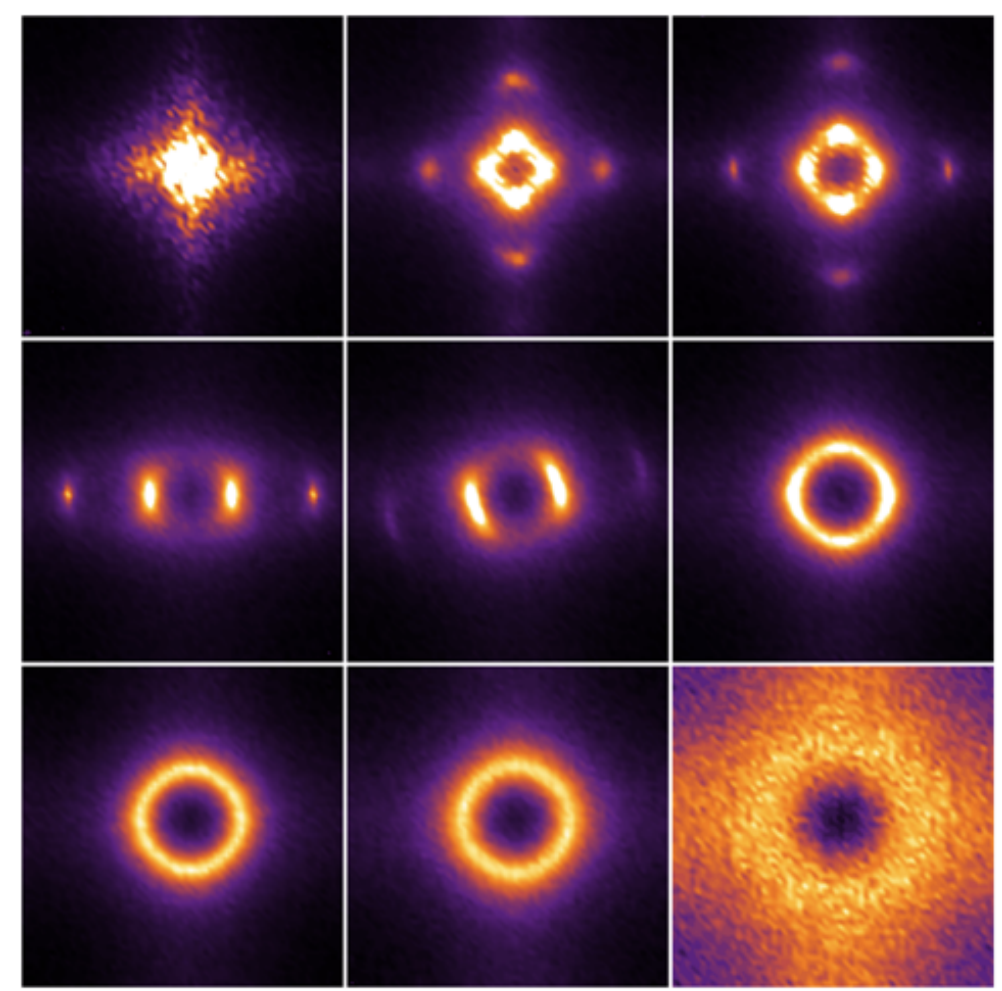

Figure 3: The Fourier transform $\tilde{\phi}(k)$ in the $d=2$ ICDW model on a $64^{2}$ lattice for several values of $g$, averaged over 200 measurements. From left to right, top to bottom: $g=0.8,0.9,1.0,1.1,1.2,1.3,1.4,1.5$ and 2.5. The other parameters are $m_{\chi}^{2}=0.5, \lambda=0.1$ and $v=3$.

Our final two-component model, the Bose gas at finite density, is different from the first three because the imaginary part of the action is associated with the kinetic term in the action. The Bose gas at finite density has the same anti-linear symmetry as QCD: the PT symmetry is actually $C K$. The complex field $\Psi$ can be decomposed as $\Psi=\frac{1}{\sqrt{2}}(\phi+i \chi)$. At zero chemical potential, this model is invariant under $\mathscr{C}$ and $\mathscr{K}$ separately but for chemical potential $\mu$ nonzero, temporal derivative terms are complex and the Lagrangian is only $\mathscr{C} \mathscr{K}$ symmetric: $\mathscr{L}(\phi, \chi)^{*}=\mathscr{L}(\phi,-\chi)$. The action is given by

$$
S(\chi)=\sum_{x}\left[\left(e^{\mu} \Psi^{*}(x+\hat{t})-\Psi^{*}(x)\right)\left(e^{-\mu} \Psi(x+\hat{t})-\Psi(x)\right)+\frac{1}{2}|\nabla \Psi(x)|^{2}+m^{2}|\Psi(x)|^{2}\right]
$$

After a series of Fourier transforms to eliminate $\chi$, the action $\tilde{S}$ takes the form

$$
\tilde{S}=\frac{1}{2} \cosh \mu\left(\partial_{4} \phi\right)^{2}+\frac{1}{2}(\nabla \phi)^{2}+\frac{1}{2} \pi_{\mu}^{2}+\tilde{V}\left(\phi, \pi_{\mu}\right)
$$

where

$$
\tilde{V}\left(\phi, \pi_{\mu}\right)=\frac{1}{2} m^{2} \phi^{2}+\frac{1}{2 M^{2}}\left(\cosh \mu\left(\partial_{4} \pi_{4}\right)+\nabla \cdot \vec{\pi}-\sinh \mu\left[\phi\left(x+\hat{e}_{4}\right)-\phi\left(x-\hat{e}_{4}\right)\right]\right)^{2}
$$


with the mass parameter $M$ determined by $M^{2} \equiv 2\left(1+\frac{1}{2} m^{2}-\cosh \mu\right)$. The action is positive only if $M^{2}>0$. This is the lattice form of a familiar condition for scalar theories at finite density, analogous to $m \leq \mu$ in the continuum. The restriction to $M^{2}>0$ poses potential difficulties for an interacting theory with $m^{2}<0$. A dual representaton can be constructed for $m^{2}<0$ using a polar decomposition of the fields [10].

\section{The case of $i \phi^{3}$ transitions}

Models in the $i \phi^{3}$ universality class are problematic because their partition functions cannot be everywhere positive near an isolated Lee-Yang zero. This directly implies that there can be no representation of $Z$ which is everywhere positive near such a zero. The original case of the Ising model in an imaginary field is instructive: the partition function must vary in sign as LeeYang zeros are crossed. According to the Lee-Yang circle theorem, such a zero must lie on the line $\operatorname{Re}(h)=0$, and $P T$ symmetry tells us that $Z$ is real on this line. Because $Z$ is positive when $\operatorname{Im}(h)=0$, the partition function $Z$ must be negative along $\operatorname{Re}(h)=0$ between the first and second zero above the origin. This behavior has a natural explanation using $P T$ symmetry: zeros arise when the largest eigenvalues of the transfer matrix $T_{t}$ form a conjugate pair. If we suppose that the partition function can be written as $Z=\operatorname{Tr}\left(T_{t}^{N}\right)$ where $N$ is the extent of the system in some direction, then $P T$ symmetry gives a representation of $Z$ as

$$
Z=\sum_{p}\left(\lambda_{p}^{N}+\lambda_{p}^{* N}\right)+\sum_{r} \lambda_{r}^{N}
$$

where the sums are over all complex pairs $p$ of eigenvalues of $T$ and all real eigenvalues $r$. In the case where $N$ is large and the partition function is dominated by a largest pair of complex eigenvalues, $Z$ is approximately

$$
Z \approx \lambda_{0}^{N}+\lambda_{0}^{* N}=\left|\lambda_{0}\right|^{N} 2 \cos \left(N \phi_{0}\right) .
$$

This indicates that $Z$ will oscillate between positive and negative values as $N$ and $\phi_{0}$ vary whenever a complex pair of eigenvalues of $T$ dominates the partition function. A similar point of view on the relevance of Lee-Yang zero has recently been given in [11]. There are several important $P T$-symmetric models which contain within them forms of the $i \phi^{3}$ transition as the parameters of the model are varied. Typical examples of such models are $Z(3)$ or $S U(3)$ spin systems which are strong-coupling approximations to lattice QCD at nozero temperature and density. In $Z(3)$, for example, the group elements are the set $\{1,(-1+i \sqrt{3}),(-1-i \sqrt{3})\}$. An additional term in the lattice action which favors the second two elements can lead to a phase transition in the $i \phi^{3}$ universality class.

\section{Conclusions}

There is a simple, local algorithm that allows for simulation of a large class of scalar models with complex actions. This provides benchmark results for proposed sign problem algorithms. Simulation indicates that pattern formation may be a common feature of models with sign problems and a phase transition, occurring when a tachyonic instability of the global translation-invariant 
phase is present. The ICDW model has several features in common with QCD at finite density. If we identify $\phi$ with the real part of the Polyakov loop and $\chi$ with the imaginary part, then the ICDW model as $g$ is varied is similar to motion along the critical line of finite density QCD. This suggests that there is a region of parameter space where pattern formation occurs in models of finite density QCD. Unfortunately, the presence of an $i \phi^{3}$ transition embedded within a particular model's parameter space is an obstacle to a successful transformation of many important sign problem models into a manifestly positive form.

\section{References}

[1] P. N. Meisinger and M. C. Ogilvie, PT Symmetry in Classical and Quantum Statistical Mechanics, Phil. Trans. Roy. Soc. Lond. A 371, 20120058 (2013) [arXiv: 1208.5077 [math-ph] ].

[2] C. M. Bender and H. F. Jones, Interactions of Hermitian and non-Hermitian Hamiltonians, J. Phys. A 41, 244006 (2008) [arXiv:0709.3605 [hep-th]].

[3] C. M. Bender, A. Felski, N. Hassanpour, S. P. Klevansky and A. Beygi, Analytic structure of eigenvalues of coupled quantum systems, Phys. Scripta 92, no. 1, 015201 (2017) [arXiv:1702.03839 [math-ph]].

[4] Michael Seul and David Anderlman, Domain Shapes and Patterns: The Phenomenology of Modulated Phases, Science 267, 476 (1995).

[5] Z. Nussinov, J. Rudnick, S. Kivelson and L. N. Chayes, Avoided Critical Behavior in O(n) Systems, Phys. Rev. Lett. 83, 472 (1999)

[6] Saurish Chakrabarty and Zohar Nussinov, Modulation and correlation lengths in systems with competing interactions, Phys. Rev. B 84, 144402 (2011) [arXiv:0906.5381 [cond-mat].

[7] D. G. Ravenhall, C. J. Pethick and J. R. Wilson, Structure Of Matter Below Nuclear Saturation Density, Phys. Rev. Lett. 50, 2066 (1983).

[8] M. Hashimoto, H. Seki and M. Yamada, Shape of Nuclei in the Crust of Neutron Star, Prog. Th. Phys. 71, 320 (1984).

[9] C. J. Horowitz and D. K. Berry, The Shear Viscosity and Thermal Conductivity of Nuclear Pasta, Phys. Rev. C 78, 035806 (2008) [arXiv: 0807.2603 [astro-ph]].

[10] C. Gattringer and T. Kloiber, Lattice study of the Silver Blaze phenomenon for a charged scalar $\phi^{4}$ field, Nucl. Phys. B 869, 56 (2013), [arXiv: 1206.2954 [hep-lat] ].

[11] P. de Forcrand and T. Rindlisbacher, Spin models in complex magnetic fields: a hard sign problem, EPJ Web Conf. 175, 07026 (2018) [arXiv:1711.00042 [hep-lat]]. 